\title{
Permuta Financiera SWAP
}

${ }^{(1)}$ Daniel Mavila Hinojoza

${ }^{(2)}$ Antonio Luyo Quiroz

\begin{abstract}
RESUMEN
El manejo de la gestión de riegos de mercado se puede realizar a través de los llamados productos derivados con fines de cobertura; la permuta financiera (swap) es uno de los instrumentos financieros a disposición del gerente financiero y que es materia del presente artículo; asimismo, se presentan casos aplicativos para que la empresa mitigue la posibilidad de que sufra pérdidas debido a movimientos inopinados y adversos en los tipos de interés, tipo de cambio, precios bursátiles y de las materias primas o commodities.
\end{abstract}

Palabras clave: Permutas financieras; derivados; ingeniería financiera.

\section{SWAP}

\section{ABSTRACT}

The handling of market risk management can be done through derivative instruments for hedging purposes. The swap transaction is one of the financial instruments available to the financial manager and that is the subject of this article. Also application cases are appearing for the company mitigates the possibility of suffering losses due to unexpected and adverse movements in interest rates, exchange rates, stock prices and raw materials or commodities.

Key words: Swaps; derivatives; financial engineering.

\section{INTRODUCCIÓN}

Muchos de los riesgos a los que se enfrenta la empresa moderna con la globalización de la economía le son inherentes al desarrollo de su propia actividad productiva y son los llamados riesgos de negocio, empresariales o económicos vinculados a la elaboración y comercialización de los productos y servicios de la compañía.

Adicionalmente, las empresas se encuentran sometidas a otros riesgos de origen financiero, que cada vez ejercen una mayor influencia sobre estas. Para el efecto, desde la década de los setenta del siglo pasado, se empezaron a desarrollar nuevas técnicas de gestión de riesgos que han devenido en la creación de los instrumentos financieros derivados.

Adecuadamente utilizados, los productos derivados permiten transferir los riesgos permitiendo anticiparse a las consecuencias favorables o desfavorables de las variables del negocio, limitando de esta manera las pérdidas potenciales, estabilizar los flujos de caja, además de aportar flexibilidad, rapidez y bajos costos de transacción.

\section{INSTRUMENTOS FINANCIEROS DERIVADOS: SWAPS}

Los instrumentos financieros derivados son llamados así porque su precio deriva del valor de otro activo (divisas, bonos financieros, materias primas, etc.), el que es denominado activo subyacente.

Por ejemplo, en un forward, la ejecución del contrato no se realiza en el momento que se suscribe el mismo, sino en una especificada fecha de vencimiento. En la fecha en que se formaliza, las partes contratantes acuerdan los términos del contrato, incluido el precio a plazo o precio forward de la operación [1].

Estos contratos, en general, se pueden realizar en mercados organizados como, por ejemplo, NYMEX (New York Mercantile Exchange), o en mercados no organizados usualmente conocidos como Over The Counter (OTC), que es el caso peruano.

1 Magíster. Docente Principal. Facultad de Ingeniería Industrial. Universidad Nacional Mayor de San Marcos. E-mail: danielmavila@yahoo.es

2 Doctor. Docente Asociado. Facultad de Ingeniería Geológica, Minera, Metalúrgica y Geográfica. Universidad Nacional Mayor de San Marcos. E-mail: aluyoq@unmsm.edu.pe 
En los mercados derivados no organizados, conocidos también como mercados de mutuo acuerdo, se transan operaciones a plazo, opciones OTC y operaciones de permuta financiera o swaps, siendo la aplicación en el caso peruano para coberturarse, generalmente, de las variaciones en los tipos de cambio de la divisa, tipos de tasas de interés y precio de las materias primas.

EI ISDA (International Swap and Derivatives Association) es la asociación norteamericana que ha desarrollado modelos normalizados de operaciones financieras de cobertura con base a su experiencia en este tipo de transacciones en que existe un componente transnacional. Las ventajas de utilizar estos acuerdos marcos se derivan precisamente de la estabilidad y seguridad que proporciona su carácter estandarizado.

Es así que el ISDA Master Agreement ${ }^{[2]}$ es un acuerdo marco normalizado que se utiliza de manera habitual en operaciones financieras con instrumentos derivados en los mercados internacionales ${ }^{[3]}$, el cual consiste en una serie de documentos que definen todos y cada uno de los términos empleados. Los bancos locales le agregan a este acuerdo marco anexos de condiciones particulares, confirmaciones escritas de cada una de las transacciones que, en general, se conciertan por teléfono o vía Internet, así como todo un conjunto de normas y principios contenidos en las Definiciones de ISDA (ISDA Definitions).

EI ISDA señala que el mercado de derivados OTC, medido en función de montos nacionales, alcanzó US $\$ 450,4$ billones de dólares americanos, de los cuales US $\$ 38,6$ billones corresponden a derivados de crédito (ISDA 2008) ${ }^{[4]}$. Estas cifras aparentan exageración; sin embargo, se debe considerar que el mercado de los derivados OTC tiene un apalancamiento de casi siete veces el producto interno bruto del mundo.

\section{OPERACIONES DE PERMUTA FINANCIERA}

La palabra inglesa swap significa: intercambio, trueque; siendo los contratos más resaltantes los de intercambios de intereses (Interest Rate Swaps) y los SWAPS de Divisas (Cross Currency Swaps), los cuales se analizarán a continuación:

\section{LOS SWAPS DE INTERESES (IRS)}

Son contratos que obligan a las partes a intercambiar, en fechas pre-establecidas, los intereses correspondientes al aplicar dos tipos de interés diferentes a un capital nocional (Valor nominal de referencia sobre el cual se calculan los cobros y pagos futuros) acordado. Los contratos de swaps de intereses no incluyen el intercambio de capitales o principal, como sí ocurre con los swaps de divisas.

La mayoría de los swaps de intereses se basan en el intercambio de los intereses correspondientes a una tasa fija, por los correspondientes a una tasa de interés flotante (Limabor, Libor, Prime rate, etc.). Por ello, este tipo de swap se conoce en inglés como plain vanilla, es decir "simple vainilla", por comparación con el tipo de helado más sencillo de producir comercialmente.

Los swaps de intereses surgieron debido al hecho que, aunque muchos grandes bancos podían tomar dinero prestado a largo plazo a tasas fijas en mejores condiciones que muchas empresas, no deseaban mantener compromisos con intereses fijos a largo plazo, cuando sus depósitos estaban sujetos a intereses variables a corto plazo (generalmente, Libor de 90 días).

Por otra parte, muchas empresas industriales podían conseguir préstamos en base Libor en muy buenas condiciones, pero preferían tener tasas de interés fijas evitando de esta manera correr riesgos de subidas de intereses. El mercado de swaps de intereses se desarrolló muy rápidamente en Londres y Nueva York a partir de 1982. Inicialmente, los márgenes eran muy grandes, y ello atrajo a muchos partícipes, incluyendo intermediarios especializados, los swap brokers (agentes de swaps) y swap dealers (intermediarios de swaps que actúan también como contrapartida en este tipo de derivados).

Si bien los swaps de tipos de interés son instrumentos utilizados principalmente por entidades financieras e industriales; hoy en día, muchos bancos los han hecho accesibles a sus clientes individuales como es el caso del BBVA (Banco Continental), BCP (Banco de Crédito del Perú), entre otros. En estos casos, generalmente, el cliente suele tomar el interés fijo y pagar el interés flotante sobre un capital nocional, depositando un porcentaje de ese capital (10 al 15\%) en garantía de cumplimiento.

La operatividad de esta transacción es equivalente a comprar un activo de renta fija y financiar su compra con un préstamo a corto plazo que se va renovando. Así, mientras las tasas de interés a largo plazo se mantengan por encima del interés a corto, el inversor puede conseguir rendimientos muy importantes en términos del capital aportado. 
Cuadro 1. Flujos del IRS variable por fija

\begin{tabular}{|c|c|c|c|c|}
\hline Año & $\begin{array}{c}\text { LIMABOR } \\
\text { (supuesto) }\end{array}$ & $\begin{array}{c}\text { Flujos a tipo } \\
\text { Fijo (6\%) pagados } \\
\text { por A }\end{array}$ & $\begin{array}{c}\text { Flujos a tipo Variable } \\
\text { pagados por el } \\
\text { Banco BBVA }\end{array}$ & $\begin{array}{c}\text { Flujo de caja } \\
\text { neto (netting) } \\
\text { nuevos soles }\end{array}$ \\
\hline 1 & $5,5 \%$ & 60000 & 55000 & -5000 \\
2 & $6,5 \%$ & 60000 & 65000 & +5000 \\
3 & $7,0 \%$ & 60000 & 70000 & +10000 \\
\hline
\end{tabular}

Fuente: Elaboración propia.

Sin embargo, en los casos de subidas de tipos a corto por encima de los intereses a largo plazo, las pérdidas son igualmente importantes. En definitiva, para el inversor particular, esta fórmula de inversión en swaps de intereses, no es sino un formato más de inversión apalancada, que conlleva los altos niveles de riesgo asociados con esas inversiones.

\section{CASO PRÁCTICO DE IRS}

La empresa constructora A pacta un préstamo por $\mathrm{S} /$. 1 millón de nuevos soles a una tasa variable LIMABOR + 0,5\% de comisión, con pago de intereses al final de cada año y cancelación del principal al final del tercer año. A fin de convertir su deuda a tasa fija y poder trasladar el gasto financiero fijo a sus potenciales compradores, que es el estándar en la venta de inmuebles, contrata con el Banco BBVA un swap de 1 millón de nuevos soles a tasa fija de $6 \%$ anual.

Mediante el swap, la empresa A le abonará anualmente al BBVA los intereses a tasa fija de $6 \%$ de su deuda a cambio de recibir los intereses a tipo variable (LIMABOR). El resultado neto será que A queda endeudada a tipo fijo tal como se deseaba, los cálculos se muestran en el Cuadro 1.

En este caso, el beneficiado por el swap ha sido el pagador a tipo fijo, o sea, la empresa A; ya que los tipos han subido más de lo que esperaba el pagador a tipo variable, el BBVA, quien a su vez habrá efectuado otras transacciones contrarias que, finalmente, como institución bancaria le permitan asegurar sus ganancias como intermediario financiero.

Valga anotar que en estas operaciones, la obligación de pago se refiere exclusivamente al intercambio de intereses, siendo el principal utilizado para calcular los intereses respectivos. Es por esta razón que estas transacciones no tienen impacto en los balances contables de las empresas participantes, solo afectan al estado de ganancias y pérdidas, por lo que son clasificadas como instrumentos fuera de balance. Los pagos de ambas partes se realizan por compensación de la diferencia, conocida como netting.

\section{SWAP DE DIVISAS (CURRENCY SWAP)}

Constituyen un intercambio de principales e intereses de préstamos en distintas monedas. A su vencimiento, se produce el intercambio de los principales al tipo original. De tal manera que los flujos de intereses se calculan con referencia a los tipos fijos, conociéndose esta operación como plain vanilla currency swap.

\section{CASO PRÁCTICO DE CURRENCY SWAP}

La empresa peruana A requiere US\$10 millones para invertirlos en EE.UU., pudiendo conseguirlos en un banco de EE.UU, en el mejor de los casos, a $8 \%$ anual por concepto de intereses. La empresa norteamericana The Best Company quiere disponer de S/. 30 millones para invertirlos en el Perú y puede conseguirlos en el mercado local a una tasa de interés de $25 \%$ nominal anual, en el mejor de los casos. Se considerará el tipo de cambio de S/. 3.00 / US\$.

Ambas empresas deciden recurrir a un swap, para aprovechar las ventajas relativas de que dispone cada uno de ellos en su mercado primario en que emiten su deuda; para el efecto realizan las siguientes operaciones:

1) La empresa $A$ emite bonos corporativos por $S /$. 30 millones con redención a 2 años y cupón semestral de $20 \%$ nominal anual.

2) The Best Company pide prestado al Morgan Chase Bank, US\$ 10 millones por 2 años a 6\% anual pagando solo los intereses anualmente y el capital en una sola armada al final del segundo año. 
Los flujos de efectivo del swap, prescindiendo de las comisiones y contribuciones de los intermediarios se muestran a continuación:

1) Si la empresa A no hubiera efectuado el swap, su flujo sería:

Flujo de caja sin swap de la empresa A (millones de US\$)

\begin{tabular}{|l|c|c|c|c|c|}
\hline \multirow{2}{*}{ Concepto } & \multirow{2}{*}{ Año 0 } & \multicolumn{2}{|c|}{ Año 1 } & \multicolumn{2}{c|}{ Año 2 } \\
\cline { 3 - 6 } & & Semestre 1 & Semestre 2 & Semestre 1 & Semestre 2 \\
\hline Recibe & 10,0 & & & & \\
\hline Paga & & & 0,8 & & 0,8 \\
\hline Cancela & & & & & 10,0 \\
\hline
\end{tabular}

2) Con el swap, el flujo de caja para A será:

Flujo de caja con swap de la empresa A (millones de US\$)

\begin{tabular}{|c|c|c|c|c|c|}
\hline \multirow{2}{*}{ Concepto } & \multirow{2}{*}{ Año 0 } & \multicolumn{2}{|c|}{ Año1 } & \multicolumn{2}{c|}{ Año 2 } \\
\cline { 3 - 6 } & & Semestre 1 & Semestre 2 & Semestre 1 & Semestre 2 \\
\hline Recibe & 10,0 & & & & \\
\hline Paga & & & 0,6 & & 0,6 \\
\hline Cancela & & & & & 10,0 \\
\hline
\end{tabular}

Como se observa, con la realización del swap, la empresa A reduce su costo en US\$ 0,2 MM (US\$ 200000 anuales).

3) Si The Best Company no hubiera efectuado el swap, su flujo sería:

Flujo de caja sin swap de The Best Company (millones de nuevos soles)

\begin{tabular}{|c|c|c|c|c|c|}
\hline \multirow{2}{*}{ Concepto } & \multirow{2}{*}{ Año 0 } & \multicolumn{2}{|c|}{ Año1 } & \multicolumn{2}{c|}{ Año 2 } \\
\cline { 3 - 6 } & & Semestre 1 & Semestre 2 & Semestre 1 & Semestre 2 \\
\hline Recibe & 30,0 & & & & \\
\hline Paga & & 1,25 & 1,25 & 1,25 & 1,25 \\
\hline Cancela & & & & & 30,0 \\
\hline
\end{tabular}

4) The Best Company, al realizar el swap, su flujo de caja será:

\section{FLUJO DE CAJA CON SWAP DE THE BEST COMPANY (MILLONES DE NUEVOS SOLES)}

\begin{tabular}{|l|c|c|c|c|c|}
\hline \multirow{2}{*}{ Concepto } & \multirow{2}{*}{ Año 0 } & \multicolumn{2}{|c|}{ Año 1 } & \multicolumn{2}{c|}{ Año 2 } \\
\cline { 3 - 6 } & & Semestre 1 & Semestre 2 & Semestre 1 & Semestre 2 \\
\hline Recibe & 10,0 & & & & \\
\hline Paga & & & 0,8 & & 0,8 \\
\hline Cancela & & & & & 10,0 \\
\hline
\end{tabular}

\section{CASO SWAP-IN DE DÓLARES}

En este caso, la parte contratante toma posesión de dólares por algún tiempo a cambio de su moneda nacional. Transcurrido el plazo, esta debe devolver los dólares y recibir la moneda local.

Un swap-in de dólares se trata de un solo contrato equivalente a dos transacciones: una al contado y otra a plazo. Por ejemplo, se compra un millón de dólares al contado y se venden a un plazo de tres meses. En el interín, se puede disponer libremente de los dólares pero se deberán devolver al finalizar el plazo.

\section{CASO PRÁCTICO DE UN SWAP-IN DE DÓLA- RES}

Un banco del Perú desea invertir US\$10 millones de dólares en notas del Tesoro USA durante 6 meses y después volver a nuevos soles, para lo cual se cuenta con los siguientes datos:

$$
\begin{aligned}
& \text { Rs }=2.50 \% \text { anual (tasa libre de riesgo Perú) } \\
& \mathbf{R D}_{\mathbf{D}}=1.50 \% \text { anual (tasa libre de riesgo USA) } \\
& \mathrm{TC}_{\mathbf{s}}=3.4783 \mathrm{~S} / . / \$ \text { (compra spot) } \\
& \mathrm{TC}_{\mathbf{s}}=3.4800 \mathrm{~S} / . / \$ \text { (venta spot) } \\
& \mathrm{TC}_{\mathbf{F}}=3.5247 \mathrm{~S} / . / \$ \text { (compra futuro } 6 \text { meses) } \\
& \mathrm{TC}_{\mathbf{F}}=3.5439 \mathrm{~S} / . / \$ \text { (venta futuro } 6 \text { meses) }
\end{aligned}
$$

Para el efecto, el banco peruano puede contratar un swap-in dólares con otro banco peruano o extranjero (swap dealer) produciéndose los siguientes flujos:

1) Inicialmente, el banco peruano recibe $S \$ 10000000$ y entrega al swap dealer:

$10000000 \$$ * $3,4800 \mathrm{~S} / . / \$=S / .34800000$ 
2) El banco peruano sabe que en seis meses tendrá: $10000000 \$^{*}\left[1+0,015^{*}(180 / 360)\right]=\$ 10075000$

3) Para congelar su ganancia en soles, el banco peruano tendría que vender estos dólares a plazo. Debido a que está contratando un swap-in, acuerda con el banco extranjero (o peruano) que este le compre los dólares al tipo de cambio futuro.

4) Por último, el banco peruano entrega al swap dealer US\$10 075000 y recibirá:

10075000 \$ * 3,5247 S/./\$ = S/.35 511353

En esta operación, la utilidad neta para el banco peruano sería (prescindiendo de los costos de transacción):

$35511353 \mathrm{~S} /$. - 34800000 S/.= S/.711353

Expresando la ganancia en términos porcentuales se tiene $2,04 \%$ en un semestre; lo que equivale a $4,08 \%$ anual, mayor que el $2,50 \%$ anual que hubiese ganado en el Perú en un depósito sin riesgo.

\section{CONCLUSIONES}

La aplicación fundamental de los productos derivados en las empresas, particularmente del swap, es la gestión de los riesgos financieros, la reducción de los costes de financiación.

Es recomendable que la empresa que pretenda operar con estos instrumentos derivados cuente con el asesoramiento jurídico profesional adecuado para conocer todas las opciones que ofrece el acuerdo marco del swap.

\section{REFERENCIAS BIBLIOGRÁFICAS}

1. Mavila, D. (2001). Productos derivados: Forward. Industrial Data. 4[1]: 17-19.

2. http://www.isda.org/publications/isdamasteragrmnt.aspx (Visitada el 12-06-2010.)

3. http://noticias.juridicas.com/articulos/ 50Derecho\%20Mercantil/200209357710810222 521.html. (Visitada El 15-06-2010.)

4. http://revistas.pucp.edu.pe/contabilidadynegocios/files/contabilidadynegocios/2-Leiva-Contabilidad-2009-2.pdf. (Visitada El 15-06-2010.) 